\title{
Jets propagation through a hadron-string medium
}

\author{
V.P. Konchakovski, ${ }^{1,2}$ E.L. Bratkovskaya, ${ }^{3,4}$ W. Cassing, ${ }^{1}$ and M.I. Gorenstein ${ }^{2,4}$ \\ ${ }^{1}$ Institut für Theoretische Physik, Universität Giessen, Germany \\ ${ }^{2}$ Bogolyubov Institute for Theoretical Physics, Kiev, Ukraine \\ ${ }^{3}$ Institut für Theoretische Physik, Universität Frankfurt, Germany \\ ${ }^{4}$ Frankfurt Institute for Advanced Studies, Frankfurt, Germany
}

\begin{abstract}
Di-jet correlations in nucleus-nucleus collisions are studied within the HadronString-Dynamics (HSD) transport approach taking into account the reaction of the medium on the jet energy loss nonperturbatively. A comparison with the STAR and PHENIX data in central $\mathrm{Au}+\mathrm{Au}$ collisions at the RHIC energy $\sqrt{s}=200 \mathrm{GeV}$ is performed differentially, i.e. with respect to correlations in azimuthal angle $\Delta \phi$ and pseudorapidity $\Delta \eta$. The HSD results do not show enough suppression for the 'awayside' jets in accordance with earlier perturbative studies. Furthermore, the 'Machcone' structure for the angle distribution in the 'away-side' jet as well as 'ridge' long rapidity correlations in the 'near-side' jet - observed by the STAR and PHOBOS Collaborations - are not seen in the HSD results, thus suggesting a partonic origin.

PACS numbers: 24.10.Lx, 25.75.-q, 25.75.Bh, 25.75.Gz
\end{abstract}

Keywords: heavy-ion collision, jets, ridge

High transverse momentum $\left(p_{T}\right)$ partons are informative probes of the high-energydensity matter created in relativistic nucleus-nucleus $(\mathrm{A}+\mathrm{A})$ collisions. These partons loose a large fraction of their energy during the early stage of $\mathrm{A}+\mathrm{A}$ collisions before hadron formation. Such an energy loss is predicted to lead to a phenomenon known as jet quenching [1 3]. The underlying jet structure for particle production at high- $p_{T}$ can be probed using di-hadron correlations, which measure the associated particle distribution in azimuthal angle $\Delta \phi$ and pseudorapidity $\Delta \eta$ with respect to the high- $p_{T}$ 'trigger' particle. The data on two-particle spectra in the high- $p_{T}$ region in $\mathrm{Au}+\mathrm{Au}$ collisions for the c.m.s. energy of the nucleon pair $\sqrt{s}_{N N}=200 \mathrm{GeV}$ can be summarized as follows: 1) strong suppression of the away-side hadrons (jet quenching) [4, 5]; 2) specific 'Mach-cone' structure in azimuthal angle $\Delta \phi$ in the region of the away-side jet [5]; 3) long-range pseudorapidity $\Delta \phi$ correlation 
('ridge') in the region of the near-side jet [6, 7].

These experimental observations have generated great interest. In particular, various theoretical models are proposed to explain the ridge phenomenon, including (a) longitudinal flow push [8], (b) broadening of quenched jets in turbulent color fields [9], (c) recombination between thermal and shower partons [10], (d) elastic collisions between hard and medium partons (momentum kick) [11], and (e) particle excess due to QCD bremsstrahlung or color-flux-tube fluctuations focused by transverse radial flow [12]. Furthermore, longrange correlations in $\Delta \eta$ might be a consequence of string-like correlation phenomena.

In order to explore especially the latter conjecture of string-like correlations, we use the microscopic HSD transport model [13 15] for the study of di-jet correlations, which employs dominantly early string formation in elementary reactions and their subsequent decay. We recall, that the HSD model allows to explore systematically the change in the dynamics from elementary nucleon-nucleon to central nucleus-nucleus collisions in a unique way without changing or introducing new model parameters. Inelastic hadron-hadron collisions with energies above $\sqrt{s} \simeq 2.6 \mathrm{GeV}$ are described by the Fritiof model [16] (including Pythia v5.5 with Jetset v7.3 for the production and fragmentation of jets [17]) whereas low energy hadron-hadron collisions are modelled in line with experimental cross sections. We stress that no explicit parton cascading is involved in our present transport calculations.

The previous HSD analysis of high- $p_{T}$ spectra in Ref. [18] includes all model details and discusses the nuclear modification factor $R_{A A}\left(p_{T}\right)$ as the function of $p_{T}$ and centrality. In the earlier studies jets were considered perturbatively and no back-coupling to the medium has been incorporated. In extension of the previous investigations we now include the full evolution of jets in the transport approach including the response of the medium, which is important as the 'Mach-cone' and 'ridge' structures are attributed to medium evolution effects due to jet-medium interactions. In the current HSD calculations we use approximately $30 \times 10^{6}$ of $\mathrm{p}+\mathrm{p}$ inelastic collision events and $0.5 \times 10^{6}$ of central $\mathrm{Au}+\mathrm{Au}$ collisions with impact parameter $b=0$.

The di-jet correlations are measured as a function of azimuthal angle $\Delta \phi$ and pseudorapidity $\Delta \eta$ between the trigger and associated particles:

$$
C(\Delta \eta, \Delta \phi)=\frac{1}{N_{\text {trig }}} \frac{d^{2} N_{a s s o c}}{d \Delta \eta d \Delta \phi},
$$

where $N_{\text {trig }}$ is the number of trigger particles. To obtain the di-jet correlations one has to 


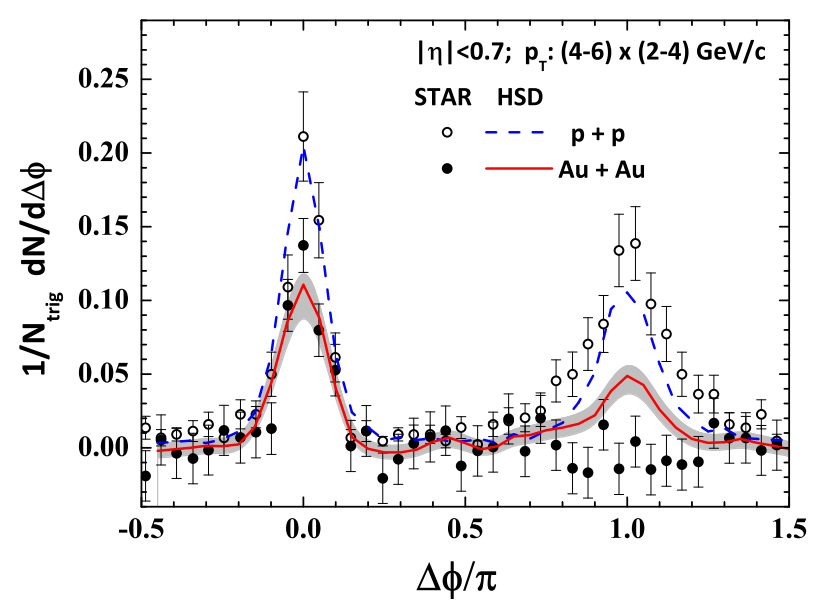

FIG. 1: Angular correlations of associated particles $\left(2<p_{T}^{\text {assoc }}<4 \mathrm{GeV} / \mathrm{c}\right)$ with respect to a trigger particle with $p_{T}^{\text {trig }}>4 \mathrm{GeV} / \mathrm{c}$ in $\mathrm{p}+\mathrm{p}$ and in central $\mathrm{Au}+\mathrm{Au}$ collision events within the HSD transport approach in comparison to the STAR data [4]. The grey area corresponds to the statistical uncertainties of the HSD calculations.

subtract a background distribution. In our calculations we use the mixed events method which allows to properly subtract the background by taking associated particles for each trigger particle from another randomly chosen event.

Angular correlations of associated particles in $\mathrm{p}+\mathrm{p}$ and central $\mathrm{Au}+\mathrm{Au}$ collisions with different cuts for $p_{T}^{\text {trig }}$ and $p_{T}^{\text {assoc }}$ are shown in Figs. 1 and 2. There are two maxima: the 'near-side' and 'away-side' peaks at $\Delta \phi=0$ and $\Delta \phi=\pi$, correspondingly. Fig. 1 provides a comaparison to the data from the STAR Collaboration with the cuts for $p_{T}^{\text {trig }}$ and $p_{T}^{\text {assoc }}$ similar to those in the previous HSD calculations [18]. We find a good agreement between the earlier perturbative [18] and current non-perturbative HSD results. Thus, one may conclude that a medium modification in this kinematic region of $p_{T}^{\text {trig }}$ and $p_{T}^{\text {assoc }}$ is small in HSD. We mention that the HSD results reasonably reproduce the data for $\mathrm{p}+\mathrm{p}$ collisions (within error bars). For $\mathrm{Au}+\mathrm{Au}$ central collisions HSD shows clearly an insufficient suppression of the 'away-side' peak at $\Delta \Phi / \pi=1$. Note also that for the most central collisions the experimentally observed suppression of single-particle spectra at high- $p_{T}$ can not fully be described by HSD: $R_{A A}^{H S D}\left(p_{T}\right)=0.35 \div 0.4$ whereas $R_{A A}^{e x p}\left(p_{T}\right)=0.2 \div 0.25$ at $p_{T}>4 \mathrm{GeV} / \mathrm{c}$. Our first conclusion is that the hadron-string medium is too transparant for high- $p_{T}$ particles (as already pionted out in [18]).

Fig. 2 corresponds to the data of the PHENIX Collaboration with different cuts for $p_{T}^{\text {trig }}$ 

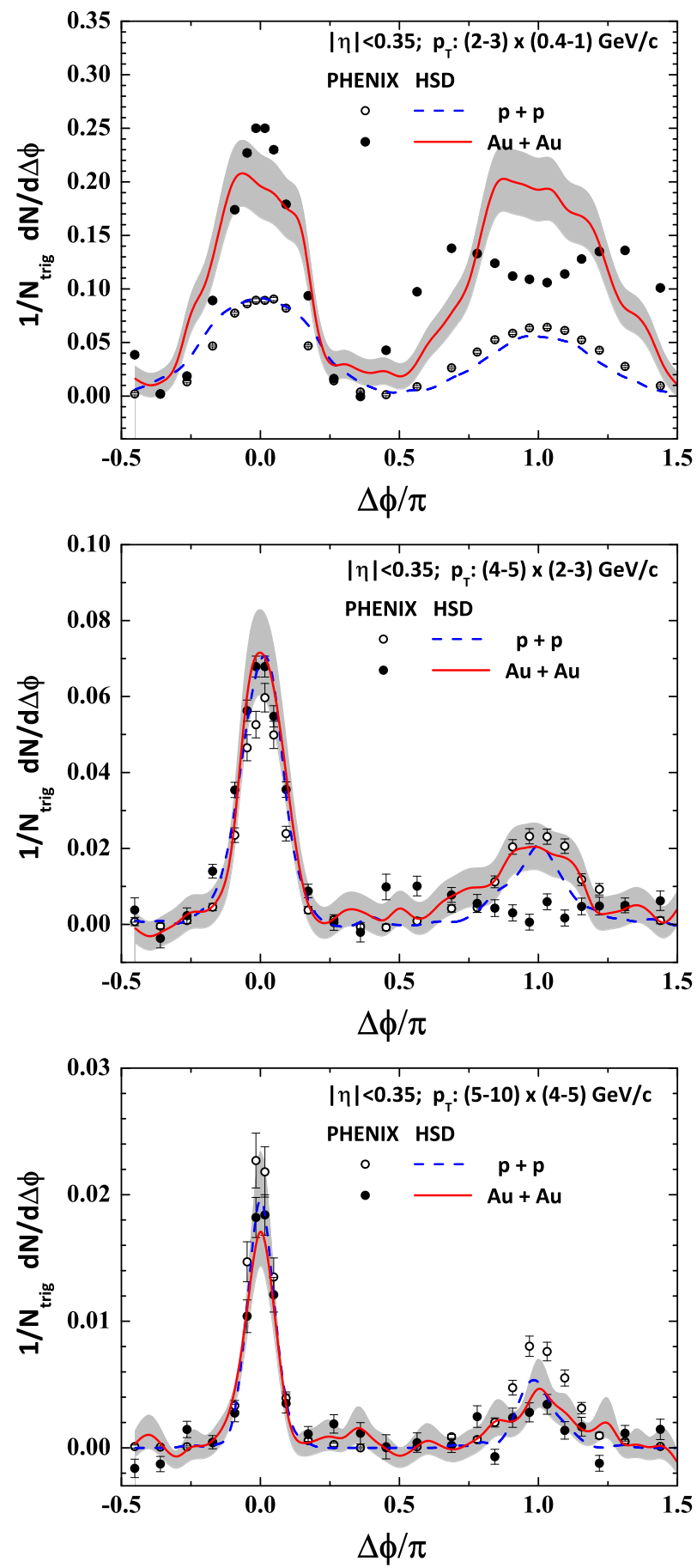

FIG. 2: Angular correlations of associated particles with different cuts for $p_{T}^{\text {trig }}$ and $p_{T}^{\text {assoc }}$ in $\mathrm{p}+\mathrm{p}$ and in central $\mathrm{Au}+\mathrm{Au}$ collisions within the HSD transport approach in comparison to the PHENIX data [5]. The grey areas correspond to the statistical uncertainties of the HSD calculations.

and $p_{T}^{\text {assoc }}$. For the top panel these cuts are $p_{T}^{\text {trig }}=2 \div 3 \mathrm{GeV} / \mathrm{c}$ and $p_{T}^{\text {assoc }}=0.4 \div 1 \mathrm{GeV} / \mathrm{c}$. This is the kinematic region where one expects a strong medium response to the jet energy 

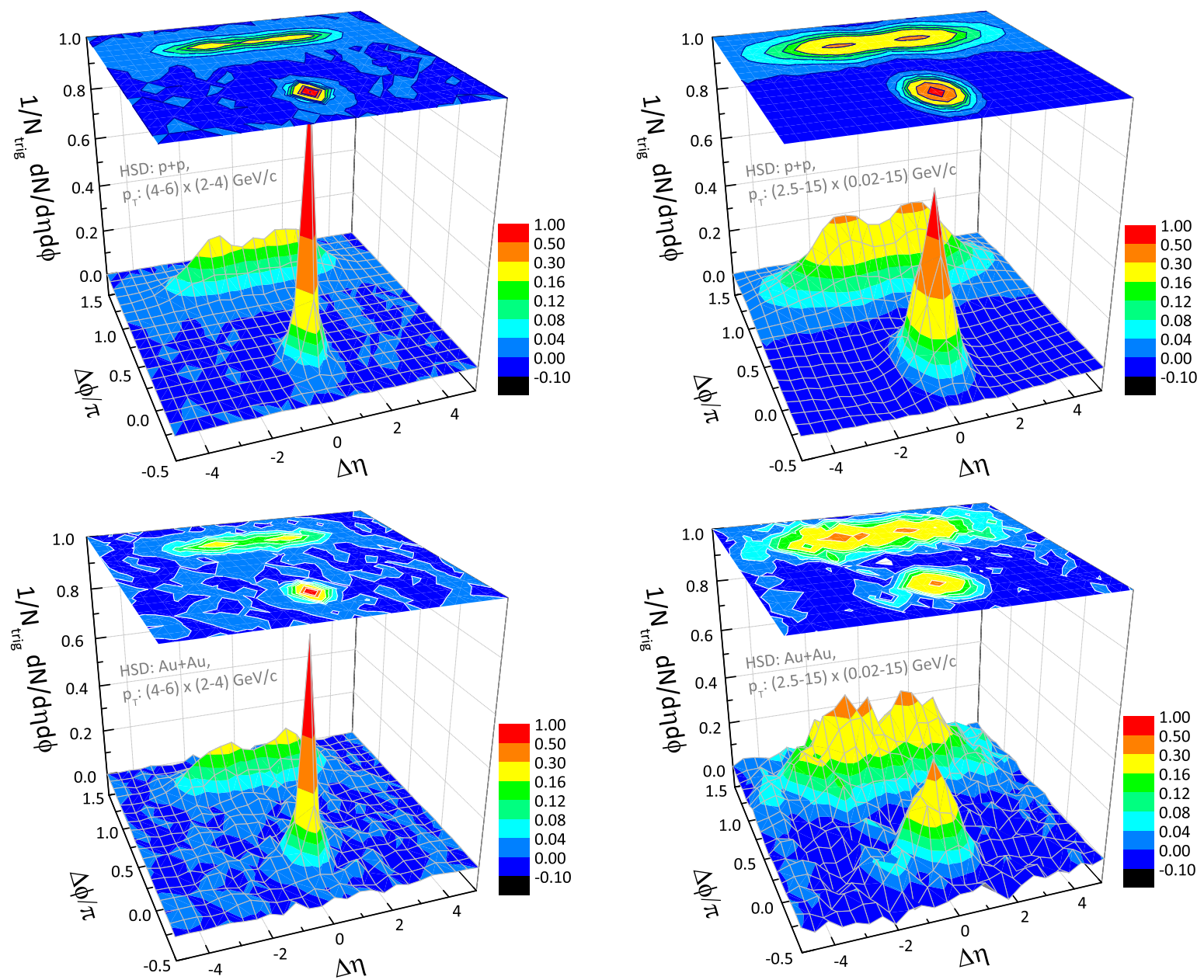

FIG. 3: The associated particle $(\Delta \eta, \Delta \phi)$ distribution (1) for $\mathrm{p}+\mathrm{p}$ (top panels) and central $\mathrm{Au}+\mathrm{Au}\left(\mathrm{b}=0\right.$, bottom panels) collisions for the trigger hadron with $4>p_{T}^{\text {trig }}>6 \mathrm{GeV} / \mathrm{c}($ left $)$ and $p_{T}^{\text {trig }}>2.5 \mathrm{GeV} / \mathrm{c}($ right) within the HSD transport approach.

loss. The experimental data show the presence of a 'Mach-cone' structure in azimuthal angle $\Delta \phi$ for the 'away-side' jet. This structure does not appear in the HSD simulations.

In Fig. 3 we present the HSD results for $\mathrm{p}+\mathrm{p}$ and $\mathrm{Au}+\mathrm{Au}$ collisions for the associated differential particle $(\Delta \eta, \Delta \phi)$ distribution (11). We use the same cuts as the STAR Collaboration, $4<p_{T}^{\text {trig }}<6 \mathrm{GeV} / \mathrm{c}$ and $2<p_{T}^{\text {assoc }}<4 \mathrm{GeV} / \mathrm{c}$ [6], and for the PHOBOS Collaboration, $p_{T}^{\text {trig }}>2.5 \mathrm{GeV}$ and $p_{T}^{\text {assoc }}>0.02 \mathrm{GeV} / \mathrm{c}$ [7]. In the HSD transport calculations we obtail on average 0.5 and 5 trigger particles in an event for the STAR and PHOBOS set of cuts, correspondingly. The di-jet correlations obtained in the HSD transport simulations of $\mathrm{Au}+\mathrm{Au}$ collisions (Fig. 3, bottom panels) do not show a ridge structure in the pseudorapidity for the near-side jet as in the data [6, 7]. 
In summary, we conclude that the HSD hadron-string medium does not show enough suppression for the nuclear modification factor $R_{A A}\left(p_{T}\right)$ at high $p_{T}$ and for the away-side jet-associated particles. For the first time the medium response on the interactions has been taken into account in the present non-perturbative HSD calculations in extenstion to previous perturbative studies [18]. The non-perturbative calculations, however, do not reproduce the experimentally observed 'Mach-cone' structure in $\Delta \phi$ for the away-side jet and the long-range rapidity correlations (the 'ridge') for the near-side jet while supporting the results from perturbative investigations. It is interesting to check in future whether the

recently proposed parton-HSD model (PHSD) [19] - incorporating explicit partonic degrees of freedom and dynamical hadronization - will be able to improve an agreement with the data and reproduce the structures observed by the PHOBOS and STAR Collaborations.

\section{Acknowledgements}

We like to thank M. Gaździcki and W. Greiner for useful discussions. This work was supported by the Helmholtz International Center for FAIR within the framework of the LOEWE program (Landesoffensive zur Entwicklung Wissenschaftlich-Ökonomischer Exzellenz) launched by the State of Hesse.

[1] R. Baier, Y. L. Dokshitzer, A. H. Mueller, S. Peigne and D. Schiff, Nucl. Phys. B 484, 265 (1997)

[2] M. Gyulassy, I. Vitev, X. N. Wang and B. W. Zhang, nucl-th/0302077.

[3] A. Kovner and U. A. Wiedemann, hep-ph/0304151.

[4] C. Adler et al. [STAR Collaboration], Phys. Rev. Lett. 90, 082302 (2003).

[5] A. Adare et al. [PHENIX collaboration] Phys. Rev. C 78, 014901 (2008).

[6] B. I. Abelev et al., [STAR collaboration] Phys. Rev. C 80, 064912 (2009); M. van Leeuwen [STAR collaboration], Eur. Phys. J. C 61, 569 (2009).

[7] B. Alver et al. [PHOBOS Collaboration], Phys. Rev. Lett. 104, 062301 (2010).

[8] N. Armesto et al., Phys. Rev. Lett. 93, 242301 (2004).

[9] A. Majumder et al., Phys. Rev. Lett. 99, 042301 (2007).

[10] C. B. Chiu and R. Hwa, Phys. Rev. C 72, 034903 (2005). 
[11] C. Y. Wong, Phys. Rev. C 78, 064905 (2008).

[12] S. A. Voloshin, Phys. Lett. B 632, 490 (2006); E. Shuryak, Phys. Rev. C 76, 047901 (2007); A. Dumitru et al., Nucl. Phys. A 810, 91 (2008); K. Dusling et al., Nucl. Phys. A 828, 161 (2009); J. Takahashi et al., Phys. Rev. Lett. 103, 242301 (2009).

[13] W. Ehehalt and W. Cassing, Nucl. Phys. A 602, 449 (1996).

[14] J. Geiss, W. Cassing, and C. Greiner, Nucl. Phys. A 644, 107 (1998).

[15] W. Cassing and E. L. Bratkovskaya, Phys. Rep. 308, 65 (1999).

[16] H. Pi, Comp. Phys. Commun. 71, 173 (1992).

[17] H.-U. Bengtsson and T. Sjöstrand, Comp. Phys. Commun. 46, 43 (1987).

[18] W. Cassing, K. Gallmeister and C. Greiner, Nucl. Phys. A 735, 277 (2004); W. Cassing, K. Gallmeister and C. Greiner, J. Phys. G 30, S801 (2004); K. Gallmeister and W. Cassing, Nucl. Phys. A 748, 241 (2005).

[19] W. Cassing and E. L. Bratkovskaya, Phys. Rev. C 78, 034919 (2008), Nucl. Phys. A 831, 215 (2009). 